\title{
Effects of strain rate on tensile deformation behaviour in Ti-6Al-4V at cryogenic temperature
}

\author{
Min-Ki Ji ${ }^{1}$, Min-Su Lee ${ }^{1}$, Yong-Taek Hyun ${ }^{2}$, Tea-Sung Jun ${ }^{1,{ }^{*}}$ \\ ${ }^{1}$ Department of Mechanical Engineering, Incheon National University, Incheon 22012, Republic of Korea \\ ${ }^{2}$ Metallic Materials Division, Korea Institute of Materials Science, Changwon 51508, Republic of Korea \\ * Corresponding author: t.jun@inu.ac.kr
}

\begin{abstract}
In this study, we investigated the effects of strain rate on tensile deformation behaviour in Ti-6Al-4V sheet at cryogenic temperature. X-ray diffraction (XRD) was used to identify the crystallographic orientation of rolled Ti-6Al-4V. A series of tensile tests were performed by constant strain rate method (CRS) with variable strain rates (i.e., on the order of $1 \times 10^{-2}$ to $10^{-4} \cdot \mathrm{s}^{-1}$ ). Liquid nitrogen (LN ${ }_{2}$ ) was used to mimic cryogenic environment, and for the thermal equilibrium the specimens were immersed in the vessel containing liquid nitrogen for $\sim 10$ minutes before tensile testing, and the temperature condition was continuously maintained during the testing. Microstructure and fracture surface was analysed by polarised light microscopy and scanning electron microscope (SEM). Electron backscatter diffraction (EBSD) was further used to characterise local deformation behaviour. Deformation twinning is occurred at cryogenic tempearture, which is rather different to the deformation at room temperature. It is thought that the twinning induced deformation behaviour may lead to a strength enhancement and a rate dependent ductility improvement.
\end{abstract}

Key words: Ti-6Al-4V, cryogenic, microstructure, deformation twinning, EBSD

\section{Introduction}

Titanium alloys, particularly $\alpha+\beta$ alloys like Ti-6Al-4V (hereinafter referred to as Ti64), have been used in machining industry owing to their promising mechanical properties. However machining using Ti64 has two problematic issues which are short tool life and rapid wear, even though processing conditions are moderate. Previous works show that cryogenic condition using liquid nitrogen as a coolant has been proposed to improve the tool life, where the tool life could be extended up to five times [1, 2], though detailed mechanisms associated with this phenomenon were not well studied. Fundamental deformation behaviour of Ti64 on extreme environment such as cryogenic temperature have been therefore issued. It has been known that dislocation plasticity is dominant deformation mechanisms at room and/or elevated temperature and twinning is typically difficult to be found in Ti64 at room temperature [3, 4]. At cryogenic temperature, however, twinning could be observed and mechanical properties are significantly different compared to those at room temperature [5]. Twinning occurred from primary (about 3\%) to secondary twinning (about $20 \%$ ) depending on local plastic strains. Because a number of slip system are limited in hexagonal close packed (HCP) metal, twinning has been considered as an essential deformation process [6, 7].

In this study, we performed a series of tensile tests of Ti64 sheet with variable strain rates at cryogenic temperature and investigated the effects of strain rate on tensile deformation behaviour through the analysis of stress-strain curve, fractography and microstructural features.

\section{Material and experiments}

Tensile specimens with gauge length of 25mm (ASTM 8M) were sectioned from rolled Ti64 sheet with thickness of $0.9 \mathrm{~mm}$, and extended along the parallel (RD) and perpendicular (TD) to the rolling directions (see Fig. 1). Crystallographic texture of Ti64 (see Fig. $2 a$ ) was analysed by a PANalytical X-ray diffraction (XRD). Table 1 shows tensile properties with respect to loading directions tested at room temperature. Since RD and TD results were almost similar in the tensile test, only TD was considered in this study.

(C) The Authors, published by EDP Sciences. This is an open access article distributed under the terms of the Creative Commons Attribution License 4.0 (http://creativecommons.org/licenses/by/4.0/). 


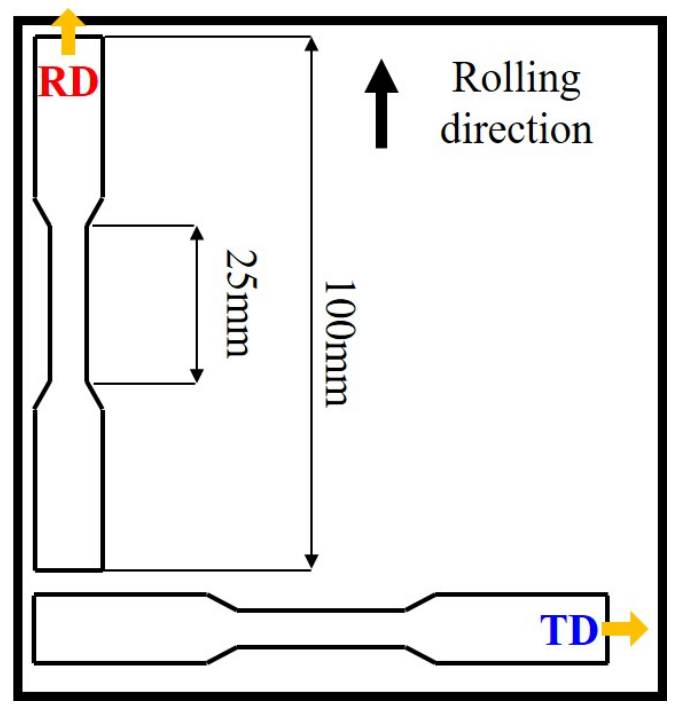

Fig. 1. The schematic diagram showing tensile specimens sectioned from rolled Ti64 sheet with thickness of $0.9 \mathrm{~mm}$.

Table 1. Tensile properties evaluated in RD and TD specimens at room temperature.

\begin{tabular}{|c|c|c|c|c|}
\hline \multicolumn{2}{|c|}{$\begin{array}{c}\text { Strain rate } \\
{\left[\mathrm{s}^{-1}\right]}\end{array}$} & $10^{-2}$ & $10^{-3}$ & $10^{-4}$ \\
\hline \multirow{2}{*}{$\begin{array}{c}\text { Yield } \\
\text { strength } \\
{[\mathrm{MPa}]}\end{array}$} & RD & $880 \pm 2.4$ & $849 \pm 1.5$ & $817 \pm 19.1$ \\
\cline { 2 - 5 } & TD & $915 \pm 5.1$ & $878 \pm 5.8$ & $844 \pm 0.7$ \\
\hline $\begin{array}{c}\text { Tensile } \\
\text { strength } \\
{[\mathrm{MPa}]}\end{array}$ & RD & $888 \pm 2.2$ & $874 \pm 2$ & $857 \pm 19.7$ \\
\cline { 2 - 5 } & TD & $915 \pm 5.1$ & $897 \pm 5.9$ & $20 \pm 1$ \\
\hline \multirow{2}{*}{$\begin{array}{c}\text { Elongation } \\
{[\%]}\end{array}$} & RD & $17 \pm 0.4$ & $18 \pm 0.9$ & $22 \pm 1.2$ \\
\cline { 2 - 5 }
\end{tabular}

The tensile test was performed by constant strain rate method until fracture at 77 and $293 \mathrm{~K}$. Strain rates of $1 \times 10^{-2}, 10^{-3}$ and $10^{-4} \cdot \mathrm{s}^{-1}$ were selected and liquid nitrogen $\left(\mathrm{LN}_{2}\right)$ was used to mimic cryogenic environment. For the thermal equilibrium the specimen was immersed in the vessel containing liquid nitrogen (see Fig. 2b) for about 10 minutes before tensile testing, and the temperature condition was continuously maintained during the testing. Note that all the specimens were fractured at the gauge length. The cross-sections located $\sim 2 \mathrm{~mm}$ below the fracture surface were ground with a series of SiC papers (up to 4000 grit) and subsequently polished with OP-S (Oxide Polishing Suspensions) diluted with $\mathrm{H}_{2} \mathrm{O}$ by a ratio 5:1 of OP-S : $\mathrm{H}_{2} \mathrm{O}$. The specimens were then etched using $2 \%$ Kroll's reagent for $\sim 20$ seconds, and a Carl Zeiss optical microscope (AXIO Scope A1) with polarised light was used to identify the microstructal features. Fracture surface was carefully observed by JEOL scanning electron microscope (SEM) and electron backscatter diffraction (EBSD) was further used to characterise local microstructure and deformation features, with an accelerating voltage of $15 \mathrm{kV}$, a probe current of $4.32 \mathrm{nA}$ and a step size of $0.25 \mu \mathrm{m}$.

(a)

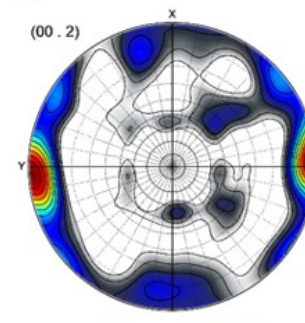

$\operatorname{Max}=3.22$

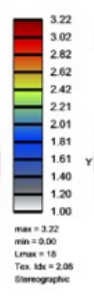

$=$

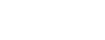

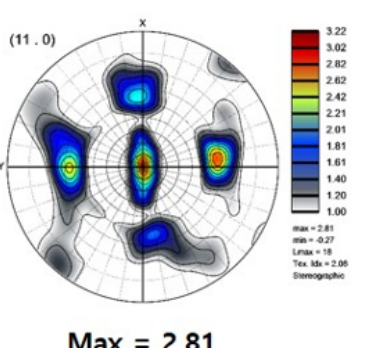

$\operatorname{Max}=2.81$

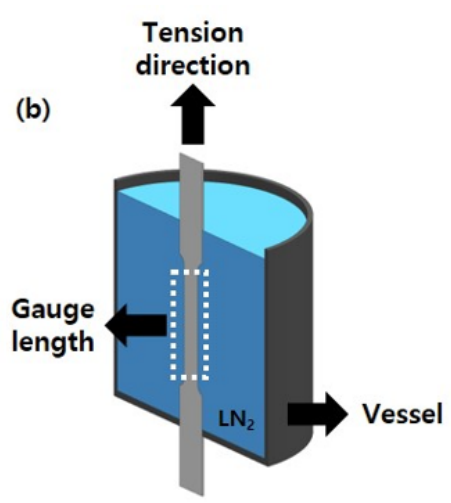

Fig. 2. (a) Pole figure (002) and (110) on Ti64 and (b) schematic diagram of tensile testing setup at cryogenic temperature.

\section{Results and discussion}




\subsection{Tensile deformation behaviour}

Fig. 3a shows engineering stress-strain curves at 77 and $293 \mathrm{~K}$ taken with variable strain rates. Tensile properties including yield strength $\left(\sigma_{\gamma}\right)$ determined by $0.2 \%$ proof stress and elongation $\left(\varepsilon_{f}\right)$ are significantly different between 77 and $293 \mathrm{~K}$. Yield strength is increased approximately $915 \mathrm{MPa}$ to $1401 \mathrm{MPa}$ but elongation decreased 17 to $10 \%$ in the strain rate of $10^{-2} \cdot \mathrm{s}^{-1}$ as temperature altered from 293 to $77 \mathrm{~K}$. Note that similar results can be found in the previous studies such that strength and hardness of Ti64 are increased while elongation is decreased at cryogenic temperature [8,9]. Fig. 3b shows yield strength and elongation distributions plotted with respect to variable strain rates. $\sigma_{Y}$ is increased as strain rate is increased, though elongation shows opposite trend at both temperatures. An interesting point here is that a ductility could be somewhat improved with controlling strain rates (i.e., lower strain rate leads to higher elongation) even at cryogenic temperature. This observation could be linked to the work by Hong et al. [1], who investigated the relationship between the tool life of Ti64 and the cutting speed under machining process with liquid nitrogen cooling. They showed that the cutting speed (i.e., 1, 1.5, 2, $2.5 \mathrm{~m} / \mathrm{s}$ ) and the associated tool life (i.e., approximately 900 , 300, 200, 50 seconds) have an inverse correlation in one nozzle method. It can be thought that Ti64 clearly possesses rate sensitive material properties at cryogenic temperature, and these properties should be considered when developing machining processes with cryogenic cooling.
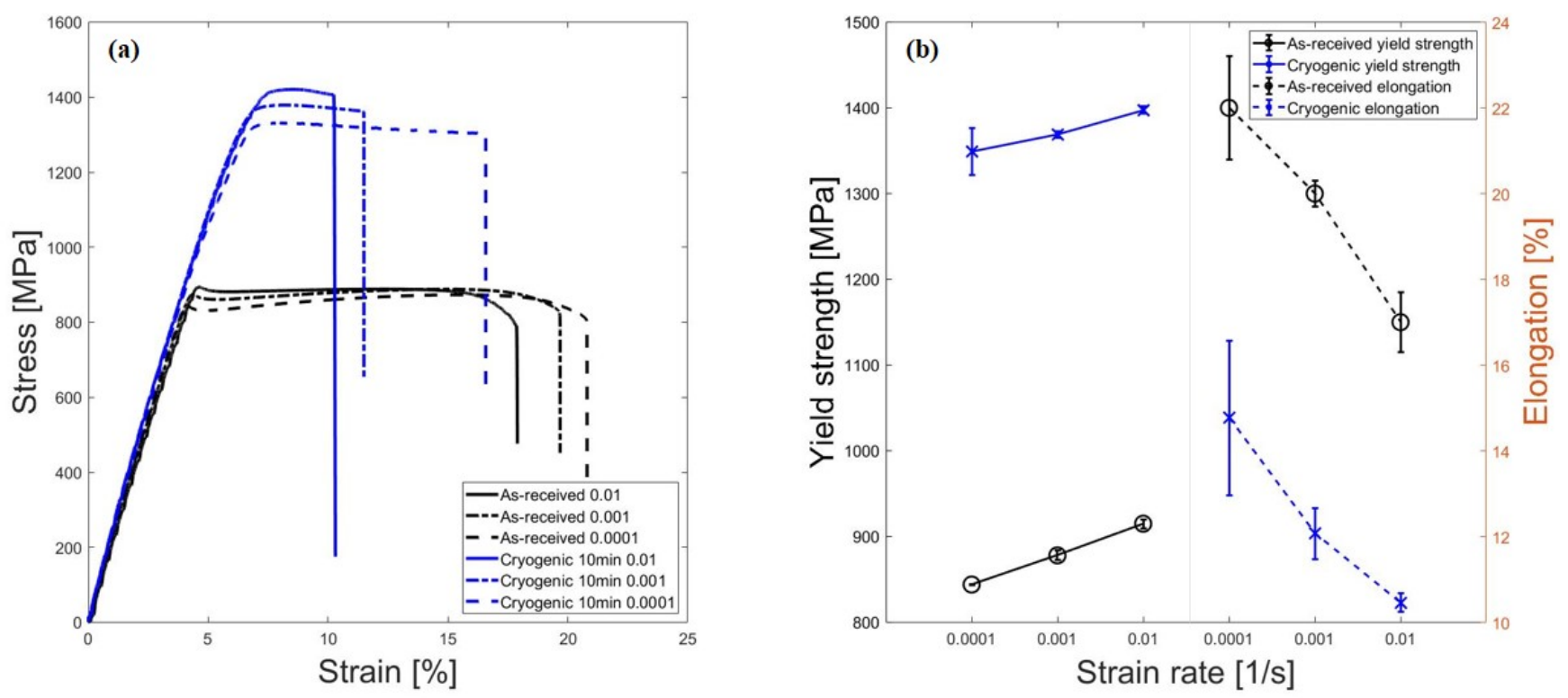

Fig. 3. (a) Engineering stress-strain curves at room and cryogenic temperature and (b) distributions of yield strength \& elongation plotted with respect to variable strain rates.

\subsection{Tension failures}

Fig. 4 shows fracture surface of Ti64 tested specimens. There are two types of fracture on fracture surface of Ti64, one is a normal failure (denoted by $\mathrm{N}$ ) and the other is a shear failure (denoted by S). Normal and shear failure is oriented at the angle of $90^{\circ}$ and $45^{\circ}$, respectively, with respect to the tension axis. Failure surfaces oriented at the angle of $45^{\circ}$ to tension axis have the dimple pattern that is elongated in the direction of the shear crack propagation (Fig. 4b), while surfaces of a normal failure have no elongation of a dimple pattern (Fig. 4a) [10]. Fig. 5 shows fracture surface at 293 and $77 \mathrm{~K}$ with variable strain rates. Normal and shear failures are present in all the fracture surface except the case for the strain rate of $10^{-4} \cdot \mathrm{s}^{-1}$ at $293 \mathrm{~K}$. At room temperature normal fracture width is increased as the strain rate gradually decreased and occurred as a whole in the case of $10^{-}$ $4 . \mathrm{s}^{-1}$ strain rate. On the other hand, shear failure is increased with decreasing strain rate and is also occured in a wide range of fracture surface in the case of $10^{-4} \cdot \mathrm{s}^{-1}$ strain rate at $77 \mathrm{~K}$. This result indicates that although a trend of mechanical responses (i.e., strength and elongation) with respect to strain rates is similar at both temperatures, the associated fracture and hence deformation behaviour is rather different. Previous study indicates that a higher ratio of " $\mathrm{N}$ " to " $\mathrm{S}$ " areas is an indication of higher ductility [11]. In the strain rate of $10^{-2} \cdot \mathrm{s}^{-1}$, this ratio is about 0.92 at $293 \mathrm{~K}$ and 0.35 at $77 \mathrm{~K}$, and the respective elongation is about $17 \%$ and $10 \%$. The ratio and elongation are increased with decreasing strain rate at room temperature. 
However in cryogenic temperature, normal fracture width and ratio are decreased while elongation is increased. This is rather different in a way that normal failure is more dominant at $293 \mathrm{~K}$ and shear failure is dominant at $77 \mathrm{~K}$ in the case of $10^{-4} \cdot \mathrm{s}^{-1}$ strain rate. Therefore, there is another apect to explain the phenomenon that lower strain rate leads to higher elongation.
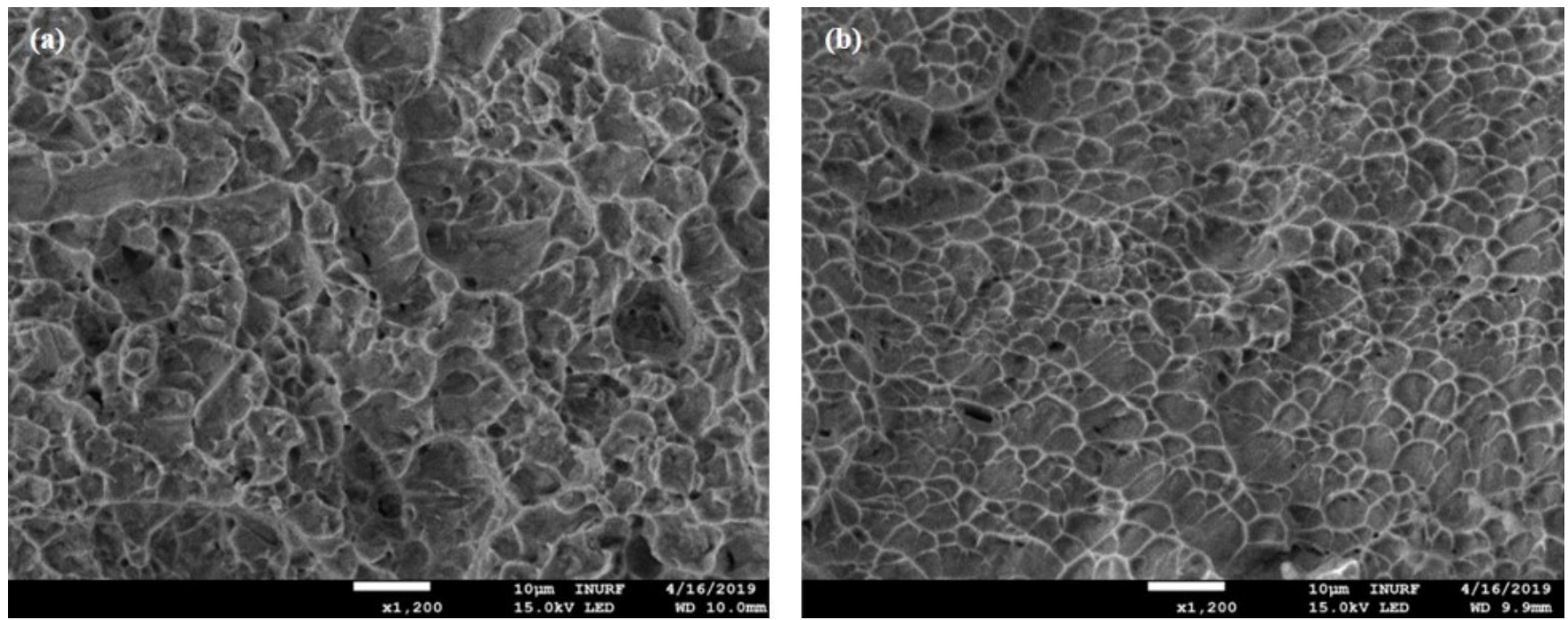

Fig. 4. Fracture surface of Ti64 tested specimens at 77 and 293K: (a) normal failure and (b) shear failure.

(a) $10^{-2} \cdot \mathrm{s}^{-1}$
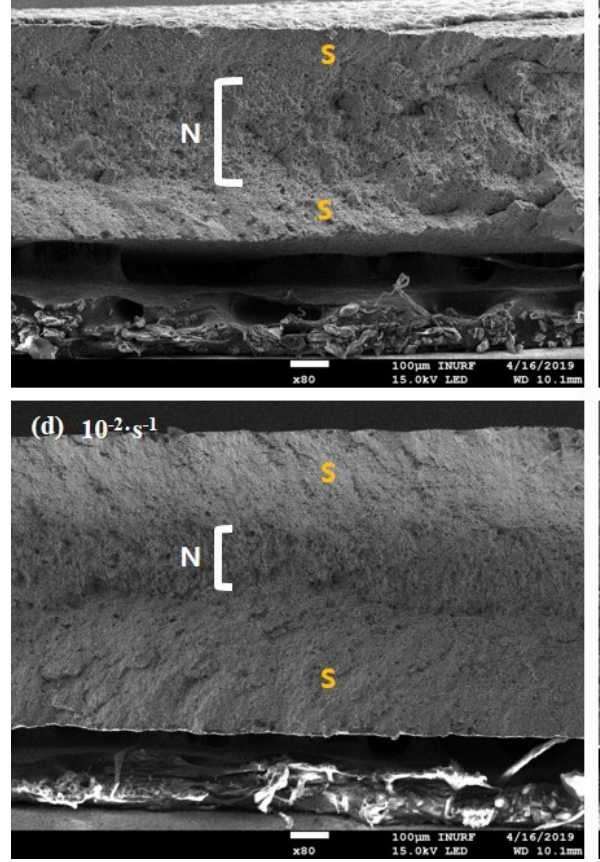

(b) $10^{-3} \cdot \mathrm{s}^{-1}$
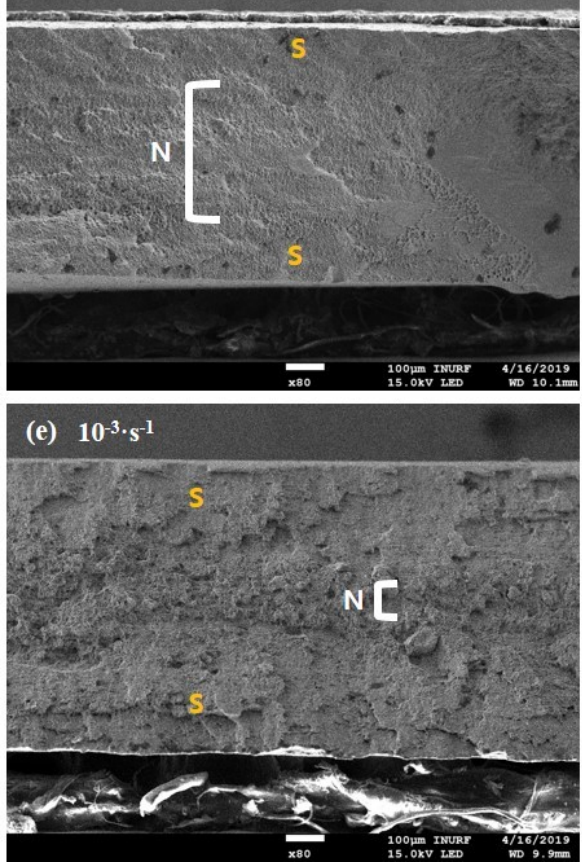

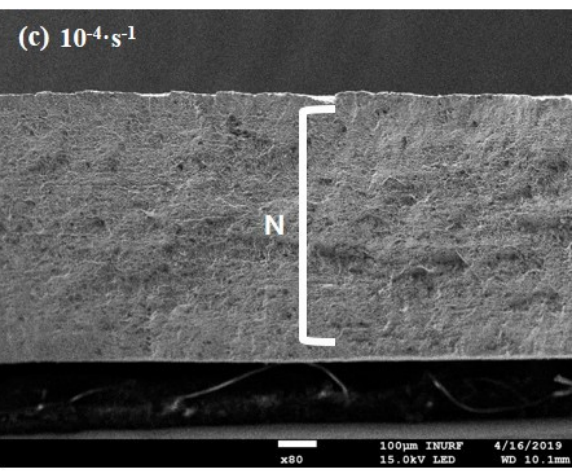

(f) $10^{-4} \cdot \mathrm{s}^{-1}$

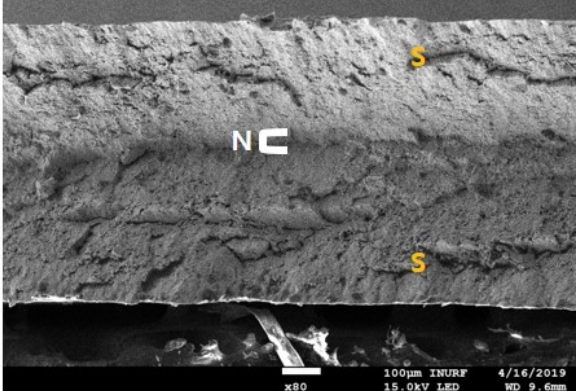

Fig. 5. Fracture surface at (a), (b), (c) $293 \mathrm{~K}$ and (d), (e), (f) $77 \mathrm{~K}$ with strain rates of $1 \times 10^{-2}, 10^{-3}$ and $10^{-4} \cdot \mathrm{s}^{-1}$, respectively.

\subsection{Deformation twinning}

Fig. 6 shows the cross-sectional area of tensile specimens below $2 \mathrm{~mm}$ from fracture point observed using a combination of differenial interference contrast (DIC) microscope and EBSD measurement. Plastic deformation is fully accomodated by slip activities at 293K, but deformation twinning is observed at 77K. It is known that the critical resolved shear stress (CRSS) for slip activation is rapidly increased as the deformation temperature is decreased, but the CRSS for twinning activation is relatively independent on the deformation temperature [12,13]. Twinning is observed regardless of the applied strain rate, though there are different trends according to locations on cross-section in Fig. 6 . In higher strain rate twinning is mostly observed at near edge, while its distribution becomes much wider as the strain rate is lowered. Fig. 7 indicates twinning area fraction calculated with respect to variable strain rates. The higher strain rate, the bigger difference the degree of twinning occurred between edge and centre zone. 
The occurrence of twinning and shear failure has similar tendency where both increases in the case of $10^{-4} \cdot \mathrm{s}^{-1}$ strain rate. Shear failure is composed of many dimples and tearing grains. At low temperatures, ductility is improved as the amount of dimples and tearing grain increases [14]. From this point of view, as twinning occurs over a wide range, shear failure also occurs as a whole, resulting in increasing elongation in case of $10^{-4} \cdot \mathrm{s}^{-1} \mathrm{strain}$ rate.

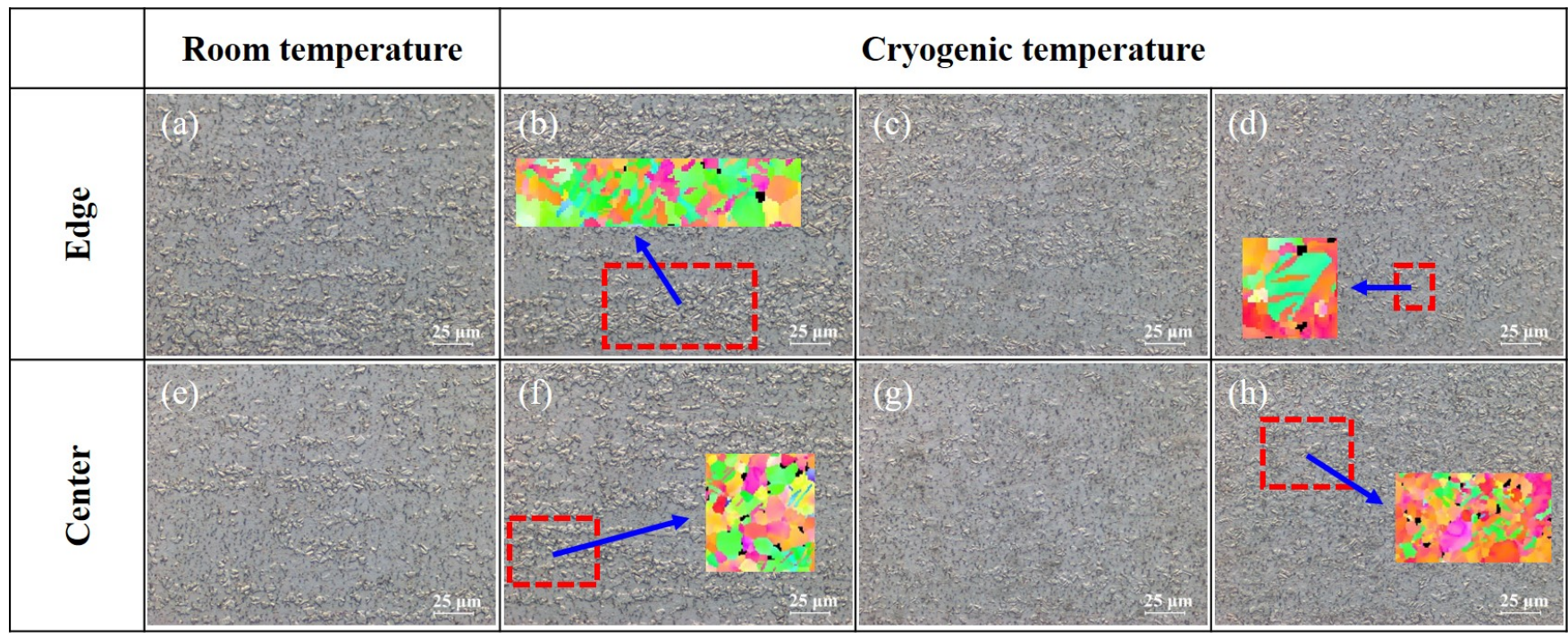

Fig. 6. Cross-sectional micrographs taken below $2 \mathrm{~mm}$ of fracture point (a) edge, (e) center regardless of strain rate at room temperature. (b), (c), (d) edge and (f), (g), (h) center with strain rates of $1 \times 10^{-2}, 10^{-3}$ and $10^{-4} \cdot \mathrm{s}^{-1}$, respectively at cryogenic temperature.
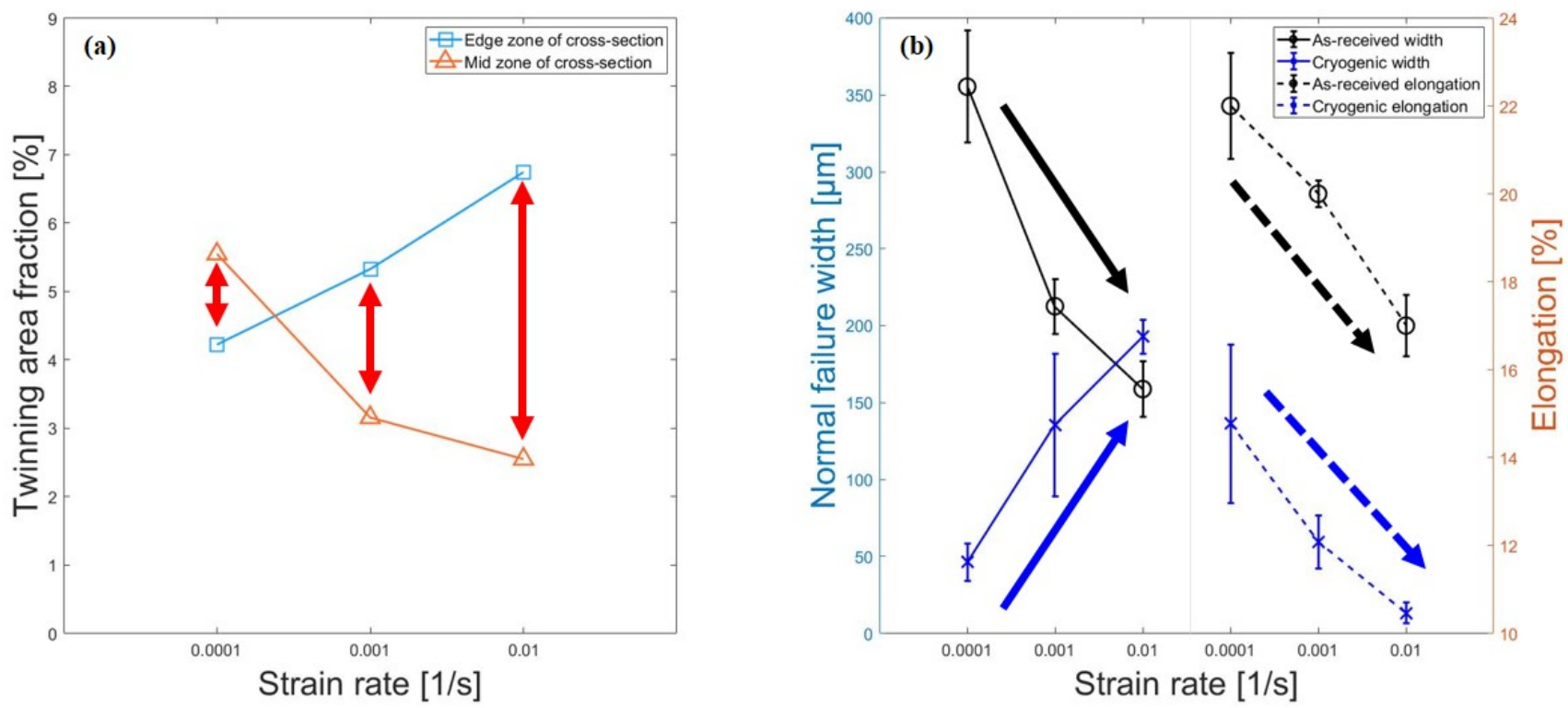

Fig. 7. (a) Twinning area fraction according to locations on cross-section and (b) normal failure width \& elongation with variable strain rates.

\section{Conclusion}

In the present study a series of tensile tests was performed at cryogenic temperature and the associated deformation mechanisms were analysed. The main results can be summarised as follows.

1. Tensile properties such as yield strength and elongation are different at $77 \mathrm{~K}$ compared to $293 \mathrm{~K}$. Yield strength and elastic range have significantly increased but elongation decreased at 77K. However, the tendency that lower strain rate leads to higher elongation is rather similar at both $293 \mathrm{~K}$ and $77 \mathrm{~K}$. 
2. There are two types of fracture regardless of strain rate and temperature, one is normal failure and the other is shear failure. As the strain rate lowered, the normal failure range increased at $293 \mathrm{~K}$ but shear failure increased at $77 \mathrm{~K}$.

3. Twinning can be found at 77K. However, the tendency of twinning occurrence according to strain rate is different. In higher strain rate twinning occurs at edge but more wider in slower strain rate. These tendency is similar to increasing shear failure in slow strain rate. So this phenomena indicates that different failure mechanism occurs at both $293 \mathrm{~K}$ and $77 \mathrm{~K}$.

\section{$\underline{\text { 5. Acknowledgements }}$}

This research was supported by Basic Science Research Program through the National Research Foundation of Korea(NRF) funded by the Ministry of Education (No. 2017R1D1A1B03033424). We would like to thank Eun-Young Kim (KIMS) for assistance in accessing EBSD.

\section{References}

[1] Hong, S.Y., I. Markus, and W.-c. Jeong, International Journal of Machine Tools and Manufacture. 41 (2001) 2245-2260

[2] Venugopal, K., S. Paul, and A. Chattopadhyay, Wear 262 (2007) 1071-1078

[3] Vanderhasten, M., L. Rabet, and B. Verlinden, Journal of materials engineering and performance 16 (2007) 208-212

[4] Roy, S. and S. Suwas, Journal of Alloys and Compounds, 548 (2013) 110-125

[5] Nayan, N., Metallurgical and Materials Transactions A 49 (2018) 128-146

[6] Yoo, M., Metallurgical Transactions A 12 (1981) 409-418

[7] Choi, S.-W., Materials Science and Engineering: A 738 (2018) 75-80

[8] Nagai, K., ISIJ International 31 (1991) 882-889

[9] Singh, G., Materials Science and Engineering: A 611 (2014) 45-57

[10] Tabachnikova, E., Rev. Adv. Mater. Sci 18 (2018) 604-607

[11] Zhirafar, S., A. Rezaeian, and M. Pugh, Journal of Materials Processing Technology 186 (2007) 298-303

[12] Williams, J., R. Baggerly, and N. Paton, Metallurgical and Materials Transactions A 33 (2002) 837-850

[13] H. Conrad, Progress in Materials Science, 26 (1981) 123-403

[14] S.L. Dong, Materials Science Forum 561-565 (2007) 207-210 